[G010]

\title{
QSRR prediction of Enantioselectivity Complex Networks related to the addition of organolithium reagents to imines in presence of chiral ligands
}

\author{
Sonia Arrasate ${ }^{1,2}$, Nuria Sotomayor ${ }^{2}$, Esther Lete ${ }^{2}$, Eugenio Uriarte ${ }^{1}$, \\ and Humberto González-Díaz ${ }^{1,3^{*}}$ \\ ${ }^{I}$ Department of Organic Chemistry, and UBICA, Institute of Industrial Pharmacy, Faculty of Pharmacy, University of Santiago de \\ Compostela, 15782,Spain, eugenio.uriarte@usc.es, humberto.gonzalez@usc.es \\ ${ }^{2}$ Department of Organic Chemistry II, Faculty of Science and Technology, University of the Basque Country/Euskal Herriko \\ Unibertsitatea, Apto.644,48080Bilbao,Spain,sonia.arrasate@ehu.es,nuria.sotomayor@ehu.es, esther.lete@ehu.es \\ ${ }^{3}$ Department of Microbiology and Parasitology, University of Santiago de Compostela, 15782, Spain.
}

\begin{abstract}
There are many enantioselective reactions of organolithium to imines described in a very different conditions. In this work, we constructed from experimental outcomes large Complex Network, which may be used to perform datamining and quantitatively describe changes in reaction variables that determine the enantiomeric excess and configuration of the stereogenic centre formed in product.
\end{abstract}

Keywords - Organolithium compounds, imines, chiral ligands, Multiple Linear Regression, QSRR, Complex Network, Molecular descriptor, Markov Model

\section{Introduction}

The asymmetric 1,2-addition of organometallic reagents to imines provides ready access to enantiomerically enriched amines with a stereogenic centre at the $\alpha$-position, an important structural feature in many biologically active compounds. ${ }^{1}$ In this kind of reactions are implicated many variables, substrates, organolithium reagents, chiral ligands, products and variables of reaction condition for instance. Therefore it exist a huge field of possible reactions to investigate.

In this sense, it is of the major interest the search of rational approaches to predict and describe the high complexity of information generated by the changes on enantioselectivity for large databases of these kind of pairs of reactions. QSRR models may be used to predict effect of changes in reaction variables over enantioselectivity but we also need tools to describe the huge amount of information generated. This sort of problem may be investigated using Complex Networks $(\mathrm{CNs})$ to regroup reactions with inverse results in which the enantioneric excess and configuration are changed from $R$ to $S$.

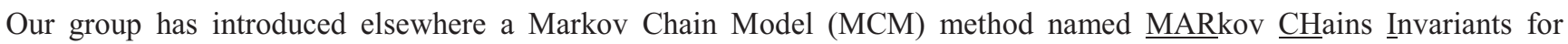
Network SImulation and Design (MARCH-INSIDE). The MARCH-INSIDE approach makes use of MCM to calculate the average values of different molecular physicochemical properties in chemical structures. ${ }^{2}$ We propose herein, for the first time, a QSRR model able to predict the difference in enantiomeric excess for $R$-product between two pair of reactions $(\Delta \mathrm{ee}(R) \%)$, which achieve to similar/dissimilar enatioselectivity after modification of reaction variables. This QSRR may predict the configuration of the new stereogenic centre formed in the synthesis of amines taking into consideration similar reaction pairs in which the enantiomeric excess increases or reduces.

\section{Results and Discussion}

We used Forward-stepwise to investigate which variables more strongly influence the change on enantioselectivity and construct the MLR-QSRR equation model. The more important variables were the differences between the initial and final reaction for: product partition coefficient $\left(\Delta \mathrm{P}_{\mathrm{p}}\right)$, chiral ligands hardness $\left(\Delta \mathrm{H}_{1}\right)$, solvent dipolar moment $\left(\Delta \mathrm{D}_{\mathrm{s}}\right)$, reaction time $\left(\Delta \mathrm{t}_{\mathrm{r}}\right)$, reaction temperature $\left(\Delta \mathrm{T}_{\mathrm{r}}\right)$, addition temperature $\left(\Delta \mathrm{T}_{\mathrm{a}}\right)$, average enantiomeric excess for reactions using same procedure $\left(\Delta \mathrm{A}_{\mathrm{e}}\right)$, substrate molar refractivity $\left(\Delta \mathrm{M}_{\mathrm{i}}\right)$, and steric constant $\left(\Delta \mathrm{S}_{\mathrm{o}}\right)$ and hardness of organolithium compounds $\left(\Delta \mathrm{P}_{\mathrm{o}}\right)$ respectively. Using these variables the best model found was: 